\title{
Nuclear Magnetic Resonance Spectroscopy
}

\author{
Teresa E. Lehmann \\ Department of Chemistry, University of Wyoming, Laramie, WY 82071, USA; tlehmann@uwyo.edu; \\ Tel.: +1-307-766-2772
}

Received: 13 April 2018; Accepted: 16 April 2018; Published: 20 April 2018

Over the past fifty years, nuclear magnetic resonance spectroscopy (NMR) has gained popularity in a wide variety of research areas. Its non-invasive character makes it ideal for the study of biomolecules and tissue samples. The possibility of determining the solution structure of molecules with the use of NMR has led to important advances in many areas including pharmacy, biology, botany, medicine, sensor design, study of polymers and more. The technology used in spectrometer and probe design have advanced to allow the study of molecules in solids, liquids, and gels. Additionally, data in the form of images, multidimensional spectra, or relaxation profiles can be obtained to study systems from different fronts. The possibility of detecting multiple nuclei allows intimate examination and characterization of a wide variety of materials.

This Special Issue devoted to NMR, and the associated Special Issue reprint, contain papers covering some of the most innovative and exiting NMR applications to different systems.

(1) Aiken, K. et al. Nuclear Magnetic Resonance Spectroscopy Investigations of Naphthalene-Based 1,2,3-Triazole Systems for Anion Sensing. Deals with the use of NMR to characterize and study the fluoride binding of 2-(4-(naphthalen-2-yl)-1H-1,2,3-triazol-1-yl)phenol (NpTP). The capability of NpTP to recognize anions makes it an asset in logic gate systems as well as molecular switches, dual detection systems, and in biological environments [1].

(2) Klemm, R. Towards a Microscopic Theory of the Knight Shift in an Anisotropic, Multiband Type-II Superconductor. In this contribution, a method is proposed to extend the zero-temperature Hall-Klemm microscopic theory of the Knight shift $\mathrm{K}$ in an anisotropic and correlated, multi-band metal to calculate $\mathrm{K}(\mathrm{T})$ at finite temperatures $\mathrm{T}$ both above and into its superconducting state. A procedure to obtain a microscopic theory of the Knight shift in an anisotropic Type-II superconductor is outlined [2].

(3) Daigle, J.-C. et al. Solid-State NMR Study of New Copolymers as Solid Polymer Electrolytes. Deals with the use of solid-state NMR to characterize comb-like copolymers, which could be used in the future in the batteries of electrical vehicles [3].

(4) Makulski, W. The Radiofrequency NMR Spectra of Lithium Salts in Water; Reevaluation of Nuclear Magnetic Moments for ${ }^{6} \mathrm{Li}$ and ${ }^{7} \mathrm{Li}$ Nuclei. In this contribution, the dipole moments of ${ }^{6} \mathrm{Li}$ and ${ }^{7} \mathrm{Li}$ were determined through NMR in the presence of dissolved ${ }^{3} \mathrm{He}$ atoms, used as a shielding reference in salt water solutions. The values obtained for the ${ }^{6} \mathrm{Li}$ and ${ }^{7} \mathrm{Li}$ magnetic moments are more reliable than those determined in water solvents [4].

(5) Brass, M. et al. Spatially Resolved Measurements of Crosslinking in UV-Curable Coatings Using Single-Sided NMR. This paper describes the extent of crosslinking in UV-curable coatings quantified through single-sided NMR. This method can be applied in evaluating systems whose crosslinking properties are intentionally varied throughout its thickness [5].

(6) Follett, S. et al. Structural Changes of $\mathrm{Zn}$ (II)bleomycin Complexes When Bound to DNA Hairpins Containing the $5^{\prime}-$ GT- $3^{\prime}$ and $5^{\prime}-$ GC- $3^{\prime}$ Binding Sites, Studied through NMR Spectroscopy. Describes the use of one- and two-dimensional NMR spectroscopy to determine the conformations adopted by $\mathrm{Zn}(\mathrm{II})$ bleomycin- $\mathrm{A}_{2},-\mathrm{A}_{5},-\mathrm{B}_{2}$, and $\mathrm{Zn}(\mathrm{II})$ peplomycin in the presence of two different 
DNA fragments. The results of the investigation indicate that the bleomycin C-termini and the DNA base sequence have an impact on the conformation of the different drugs in the target-drug complexes [6].

(7) Furihata, K. et al. Application of NMR Screening Methods with ${ }^{19} \mathrm{~F}$ Detection to Fluorinated Compounds Bound to Proteins. The NMR-based screening methods with ${ }^{19} \mathrm{~F}$-detection were applied to the human serum albumin (HSA)-diflunisal complex [7].

(8) Krishnarjuna, B. et al. Accelerating NMR-Based Structural Studies of Proteins by Combining Amino Acid Selective Unlabeling and Fast NMR Methods. Involves the application of fast NMR methods, G-matrix Fourier transform (GFT) and non-uniform sampling (NUS), to proteins samples prepared using a specific labeling scheme. The spectral simplification obtained combined with rapid data collection can help in reducing the time required for data analysis [8].

(9) Springer, T. et al. Calcium-Dependent Interaction Occurs between Slow Skeletal Myosin Binding Protein $\mathrm{C}$ and Calmodulin. The conformation of a recombinant $\mathrm{N}$-terminal fragment of slow skeletal myosin binding protein ( $\mathrm{ssC} 1 \mathrm{C} 2$ ) is characterized using differential scanning fluorimetry, nuclear magnetic resonance, and molecular modeling. The differential molecular regulation of contractility that exists between skeletal and cardiac muscle are outlined [9].

(10) Price, L. et al. Local and Average Structural Changes in Zeolite A upon Ion Exchange. Solid state NMR was used to study the changes to the local structure of the Linde Type A framework of Zeolite A. This study confirms that using a local probe such as solid state NMR alongside powder diffraction and other long-range methods to study zeolites can reveal an extra level of information about the structure of those useful minerals, which will further their use as potential catalysts and ion exchange materials [10].

(11) Cantarutti, C. et al. Short-Chain Alkanethiol Coating for Small-Size Gold Nanoparticles Supporting Protein Stability. The interaction of the 3-mercaptopropionic acid coating of AuNPs (MPA-AuNPs) with $\beta 2$-microglobulin $(\beta 2 \mathrm{~m})$ was investigated to probe protein structure perturbations. NMR and fluorescence spectroscopies were useful in determining that $\beta 2 \mathrm{~m}$ interacts with MPA-AuNPs through a highly localized patch maintaining its overall native structure with minor conformational changes [11].

(12) Vyalikh, A. et al. Early Stages of Biomineral Formation-A Solid-State NMR Investigation of the Mandibles of Minipigs. This contribution describes the changes in the mineral phase upon new tissue formation and maturation in the bone blocks surrounding dental implants in minipigs. Solid-state NMR spectroscopy allowed for the identification of inorganic species during the biomineral formation at very early stages, when crystallite particles visible in direct imaging techniques have not yet been formed [12].

(13) Yamanoi, T. et al. Separation of the $\alpha$ - and $\beta$-Anomers of Carbohydrates by Diffusion-Ordered NMR Spectroscopy. This article describes the successful use diffusion-ordered spectroscopy (DOSY) for the separation and analysis of the $\alpha$ - and $\beta$-anomers of carbohydrates with different diffusion coefficients. The individual diffusion coefficients were determined. Additionally, DOSY was also used to separate two kinds of glucopyranosides having similar aglycon structures from a mixture [13].

(14) Poirier, D. et al. NMR-Assisted Structure Elucidation of an Anticancer Steroid- $\beta$-Enaminone Derivative. The unknown product of the fortuitous modification of a quinoline-proline-piperazine side chain linked to a steroid in the presence of lithium (trimethylsilyl) acetylidethe was identified to be the $\beta$-enaminone steroid derivative through two-dimensional NMR spectroscopy [14].

(15) Nam, A.-M. et al. Quantification of Squalene in Olive Oil Using ${ }^{13} \mathrm{C}$ Nuclear Magnetic Resonance Spectroscopy. A method for direct quantification of squalene using ${ }^{13} \mathrm{C}-\mathrm{NMR}$ spectroscopy without saponification, extraction, or fractionation of the investigated samples was developed by these authors. The method led to reliable quantitative determination of squalene in olive oil samples from Corsica with an analysis time of less than three hours using a medium field NMR spectrometer (9.4 T) [15]. 
(16) Zivkovic, A. et al. Low Field NMR Determination of pKa Values for Hydrophilic Drugs for Students in Medicinal Chemistry. A bench top NMR spectrometer was used for the determination of pKa values of different drugs. The pKa values obtained showed to be in agreement with the literature data for the compounds [16].

(17) Aulikki, M. et al. The NMR ${ }^{2}$ Method to Determine Rapidly the Structure of the Binding Pocket of a Protein-Ligand Complex with High Accuracy. A NMR Molecular Replacement $\left(\mathrm{NMR}^{2}\right)$ method was developed. It is shown how $\mathrm{NMR}^{2}$ very quickly provides the complex structure of a binding pocket in a protein as measured by solution-state NMR, circumventing the long process of signal assignment [17].

(18) Proietti, N. et al. Nuclear Magnetic Resonance, a Powerful Tool in Cultural Heritage. The use of diverse NMR techniques in the monitoring and diagnosis of artworks, in order to prevent or delay their degradation, is described in this work. The development of portable NMR sensors suitable for non-destructive and non-invasive analysis in situ has made possible the investigation of precious and unmovable artefacts, and their monitoring over time [18].

(19) Porion, P. et al. Multi-Quanta Spin-Locking Nuclear Magnetic Resonance Relaxation Measurements: An Analysis of the Long-Time Dynamical Properties of Ions and Water Molecules Confined within Dense Clay Sediments. Multi-quanta spin-locking NMR relaxometry of quadrupolar nuclei was tested, in order to investigate the dynamical properties of confined fluids. This technique was shown to be a powerful tool to quantify the average residence time of molecular and ionic probes confined within the interlamellar space of clay lamellae inside dense sediments. The developed protocols are expected to be easily extended to study other interfacial systems, including porous silicate, zeolites, and cements [19].

(20) Sullivan, N. et al. Orientational Glasses: NMR and Electric Susceptibility Studies. The results of a wide range of NMR measurements of the local order parameters and the molecular dynamics of solid ortho-para hydrogen mixtures and solid nitrogen-argon mixtures are reviewed in this account. These mixtures form novel molecular orientational glass states at low temperatures. Additionally, studies of the dielectric susceptibilities of the nitrogen-argon mixtures are reviewed, in terms of replica symmetry breaking analogous to that observed for spin glass states. It is shown that this wide set of experimental results is consistent with orientation or quadrupolar glass ordering of the orientational degrees of freedom [20].

(21) Ciancaleoni, G. Characterization of Halogen Bonded Adducts in Solution by Advanced NMR Techniques. This review shows how crucial information about halogen bonding adducts can be obtained by advanced NMR techniques. It is proposed that advanced NMR techniques could be used increasingly in the near future in the consolidated, but still fruitful and rapidly evolving, field of halogen bonding in solution [21].

Conflicts of Interest: The author declares no conflict of interest.

\section{References}

1. Aiken, K.; Bunn, J.; Sutton, S.; Christianson, M.; Winder, D.; Freeman, C.; Padgett, C.; McMillen, C.; Ghosh, D.; Landge, S. Nuclear Magnetic Resonance Spectroscopy Investigations of Naphthalene-Based 1,2,3-Triazole Systems for Anion Sensing. Magnetochemistry 2018, 4, 15. [CrossRef]

2. Klemm, R.A. Towards a Microscopic Theory of the Knight Shift in an Anisotropic, Multiband Type-II Superconductor. Magnetochemistry 2018, 4, 14. [CrossRef]

3. Daigle, J.-C.; Arnold, A.A.; Vijh, A.; Zaghib, K. Solid-State NMR Study of New Copolymers as Solid Polymer Electrolytes. Magnetochemistry 2018, 4, 13. [CrossRef]

4. Makulski, W. The Radiofrequency NMR Spectra of Lithium Salts in Water; Reevaluation of Nuclear Magnetic Moments for ${ }^{6} \mathrm{Li}$ and ${ }^{7} \mathrm{Li}$ Nuclei. Magnetochemistry 2018, 4, 9. [CrossRef]

5. Brass, M.; Morin, F.; Meldrum, T. Spatially Resolved Measurements of Crosslinking in UV-Curable Coatings Using Single-Sided NMR. Magnetochemistry 2018, 4, 8. [CrossRef] 
6. Follett, S.E.; Murray, S.A.; Ingersoll, A.D.; Reilly, T.M.; Lehmann, T.E. Structural Changes of Zn(II)bleomycin Complexes When Bound to DNA Hairpins Containing the $5^{\prime}-\mathrm{GT}-3^{\prime}$ and $5^{\prime}-\mathrm{GC}-3^{\prime}$ Binding Sites, Studied through NMR Spectroscopy. Magnetochemistry 2018, 4, 4. [CrossRef]

7. Furihata, K.; Usui, M.; Tashiro, M. Application of NMR Screening Methods with ${ }^{19}$ F Detection to Fluorinated Compounds Bound to Proteins. Magnetochemistry 2018, 4, 3. [CrossRef]

8. Krishnarjuna, B.; Chandra, K.; Atreya, H.S. Accelerating NMR-Based Structural Studies of Proteins by Combining Amino Acid Selective Unlabeling and Fast NMR Methods. Magnetochemistry 2018, 4, 2. [CrossRef]

9. Springer, T.I.; Johns, C.W.; Cable, J.; Lin, B.L.; Sadayappan, S.; Finley, N.L. Calcium-Dependent Interaction Occurs between Slow Skeletal Myosin Binding Protein C and Calmodulin. Magnetochemistry 2018, 4, 1. [CrossRef]

10. Price, L.; Leung, K.M.; Sartbaeva, A. Local and Average Structural Changes in Zeolite A upon Ion Exchange. Magnetochemistry 2017, 3, 42. [CrossRef]

11. Cantarutti, C.; Bertoncin, P.; Corazza, A.; Giorgetti, S.; Mangione, P.P.; Bellotti, V.; Fogolari, F.; Esposito, G. Short-Chain Alkanethiol Coating for Small-Size Gold Nanoparticles Supporting Protein Stability. Magnetochemistry 2017, 3, 40. [CrossRef]

12. Vyalikh, A.; Elschner, C.; Schulz, M.C.; Mai, R.; Scheler, U. Early Stages of Biomineral Formation-A Solid-State NMR Investigation of the Mandibles of Minipigs. Magnetochemistry 2017, 3, 39. [CrossRef]

13. Yamanoi, T.; Oda, Y.; Katsuraya, K. Separation of the $\alpha$ - and $\beta$-Anomers of Carbohydrates by Diffusion-Ordered NMR Spectroscopy. Magnetochemistry 2017, 3, 38. [CrossRef]

14. Poirier, D.; Maltais, R. NMR-Assisted Structure Elucidation of an Anticancer Steroid- $\beta$-Enaminone Derivative. Magnetochemistry 2017, 3, 37. [CrossRef]

15. Nam, A.-M.; Bighelli, A.; Tomi, F.; Casanova, J.; Paoli, M. Quantification of Squalene in Olive Oil Using ${ }^{13} \mathrm{C}$ Nuclear Magnetic Resonance Spectroscopy. Magnetochemistry 2017, 3, 34. [CrossRef]

16. Zivkovic, A.; Bandolik, J.J.; Skerhut, A.J.; Coesfeld, C.; Raos, M.; Zivkovic, N.; Nikolic, V.; Stark, H. Low Field NMR Determination of $\mathrm{pKa}$ Values for Hydrophilic Drugs for Students in Medicinal Chemistry. Magnetochemistry 2017, 3, 29. [CrossRef]

17. Wälti, M.A.; Orts, J. The $N \mathrm{MR}^{2}$ Method to Determine Rapidly the Structure of the Binding Pocket of a Protein-Ligand Complex with High Accuracy. Magnetochemistry 2018, 4, 12. [CrossRef]

18. Proietti, N.; Capitani, D.; Di Tullio, V. Nuclear Magnetic Resonance, a Powerful Tool in Cultural Heritage. Magnetochemistry 2018, 4, 11. [CrossRef]

19. Porion, P.; Delville, A. Multi-Quanta Spin-Locking Nuclear Magnetic Resonance Relaxation Measurements: An Analysis of the Long-Time Dynamical Properties of Ions and Water Molecules Confined within Dense Clay Sediments. Magnetochemistry 2017, 3, 35. [CrossRef]

20. Sullivan, N.; Hamida, J.; Muttalib, K.; Pilla, S.; Genio, E. Orientational Glasses: NMR and Electric Susceptibility Studies. Magnetochemistry 2017, 3, 33. [CrossRef]

21. Ciancaleoni, G. Characterization of Halogen Bonded Adducts in Solution by Advanced NMR Techniques. Magnetochemistry 2017, 3, 30. [CrossRef]

(C) 2018 by the author. Licensee MDPI, Basel, Switzerland. This article is an open access article distributed under the terms and conditions of the Creative Commons Attribution (CC BY) license (http:/ / creativecommons.org/licenses/by/4.0/). 